\title{
Decisions about identity and orientation of rotated letters and digits
}

\author{
MICHAEL C. CORBALLIS, N. JANE ZBRODOFF, \\ LARRY I. SHETZER, and PATRICIA B. BUTLER \\ McGill University, Montreal, Quebec, Canada
}

In three experiments, human observers made timed decisions about alphanumeric characters, displayed singly in different orientations and versions (normal vs. backward). Latency to identify the characters was longer for backward than for normal versions, regardless of angular orientation and even under conditions in which latency was independent of angular orientation. Subjects also took longer to respond to a target orientation (whatever the character) than to respond to a target character (whatever the orientation). The results suggest that the observer first induces a description of a character that is largely independent of orientation but not of version, although the representation of version is too weak at this stage to permit an overt decision about it. Next, the angular orientation of the character is determined. Finally, the observer might "mentally rotate" the representation to the standard upright, for matching against an internally generated template.

In theories of shape recognition, it is commonly assumed that the brain extracts information about shape independently of qualities such as spatial location, size, angular orientation, and mirror reflection (e.g., Deutsch, 1955, 1962; Milner, 1974). These qualities may be described as "circumstantial;" they are not intrinsic to the shape itself, but depend rather on how it happens to be located and oriented relative to the observer. Yet there are at least some instances in which circumstantial qualities appear to provide important cues for recognition. For instance, faces are hard to recognize upside down (Rock, 1973). Again, Olshansky (Note 1; see also, Rock, 1973) showed observers various novel geometric shapes, and found that recognition was subsequently impaired if they were rotated to different angular orientations. There was very little impairment of recognition if the shapes were leftright reversed, however. Rock (1973) argued that an observer does not recognize a shape until he or she has assigned a "top" and "bottom" to it. He suggested further that, if the shape is rotated away from its customary orientation, this assignment is tantamount to an act of "mental rotation" (cf. Shepard \& Metzler, 1971). The observer "imagines" what the shape would look like in its usual orientation.

Some highly familiar shapes, such as alphanumeric characters, seem to be recognizable almost immediately

This research was supported by grants from the Defense Research Board of Canada (9425-13) and from the National Research Council of Canada. We also acknowledge the use of the computer-based laboratory of the McGill University Department of Psychology, supported by the National Research Council of Canada, the FCAC program of the Quebec Ministry of Education, and McGill University Faculty of Graduate Studies and Research. Requests for reprints should be sent to M. C. Corballis, who is now at the Department of Psychology, University of Auckland, Private Bag, Auckland, New Zealand. even if they are tilted away from the upright. This seems somewhat at odds with Rock's theory and, indeed, has been taken as evidence that recognition is based on the extraction of features which are inde. pendent of orientation (e.g., Milner, 1974). However, Rock $(1973$, p. 50) has maintained that even in this case the percept must be corrected for tilt before recognition can occur. This may be so, but one might still question whether the correction is accomplished by an act of mental rotation, as Rock proposed. Cooper and Shepard (1973) have furnished an elegant demonstration of the ability of subjects to mentally rotate the internal representations of single letters or digits, but they argue that mental rotation is not necessary for identification of a character. They required their subjects to decide as quickly as possible whether disoriented letters or digits, presented singly, were normal or backward (i.e., mirror reversed) versions. In order to perform this task, the subjects apparently did mentally rotate the characters to their standard upright positions before making their decisions. However, Cooper and Shepard imply that the subjects must have known both the identity and angular orientation of each character before mentally rotating it, for otherwise they could not have known where the upright was.

Although this conclusion seems rational on both logical and intuitive grounds, it should nevertheless be subjected to closer empirical scrutiny, for two reasons. First, it is at least conceivable (although intuitively unlikely) that subjects can mentally rotate a character in both directions at once and stop when one of the images is recognizable as a letter or digit. According to this view, mental rotation could occur prior to identification, just as Rock suggested. Second, even if Cooper and Shepard were correct in asserting that identification precedes rotation, it may still be 
the case that identification latency varies as a function of the angular orientation of the stimulus. Their latency functions might, therefore, reflect orientation-dependent processes other than mental rotation, in which case their estimates of the rate of mental rotation would require adjustment.

The general aim of the experiments reported below was to explore the sequence of operations, or stages, by which an observer comes to know the identity, angular orientation, and left-right orientation of an alphanumeric character. More specifically, we wished to discover whether identification is independent of angular orientation, as some feature-extraction theories seem to imply, or whether it is necessary to establish that a tilted character is indeed tilted before one can establish its identity. To facilitate comparison with Cooper and Shepard's (1973) results, we used the same set of stimuli, namely, the uppercase letters $G, J$, and $\mathrm{R}$, and the Arabic numerals 2, 5, and 7, presented in both forward and backward versions in angular orientations ranging from 0 to $300 \mathrm{deg}$ in 60 -deg-steps from the upright.

\section{EXPERIMENT 1}

In this experiment the subjects simply attempted to name each character as quickly as they could, and latency was recorded. Cooper and Shepard (1973) measured latency to decide whether the characters were normal or backward, and found this latency to be a steep monotonic function of the angular departure from the upright. As we have seen, they attributed this to mental rotation. However, since identification logically precedes mental rotation, we should expect latency to name the characters to be largely independent of angular orientation. We should also expect it to be independent of left-right orientation. According to the rationale of Cooper and Shepard's task, the last thing the subject knows about a character is whether it is forward or backward, and it is only following mental rotation that this knowledge is available. Prior to mental rotation, the subject should know nothing of left-right orientation, so it is reasonable to expect identification latency to be independent of it.

We might note that neither expectation is upheld in a study by Kolers and Perkins (1969), who found that the speed at which subjects could name strings of letters was significantly affected by the orientation of the letters. Letters rotated $180 \mathrm{deg}$ in their own planes took an average of $134 \mathrm{msec}$ longer per letter to name than normally oriented letters, although the difference also depended on the direction (leftward or rightward) in which the letter strings were scanned. If the letters were mirror reversed as well as rotated (which is equivalent to simple inversion), the difference jumped to $232 \mathrm{msec}$. However, these findings may not bear very closely on the interpretation of Cooper and Shepard's results, for two reasons. One is that Kolers and Perkins' subjects scanned long strings of letters, whereas Cooper and Shepard's subjects made decisions about singly presented letters; lateral scanning was clearly an important component of performance in the one case but not in the other. The second is that Kolers and Perkins included the lowercase letters $b$, $\mathrm{d}, \mathrm{p}$, and $\mathrm{q}$ in their letter strings, so that left-right orientation was critical to correct identification. Indeed, one might predict from Cooper and Shepard's results that subjects would have to perform mental rotations to tell these letters apart. This was not true of the characters used in this or in Cooper and Shepard's study.

\section{Method}

Subjects. Eight undergraduates, six women and two men, volunteered as subjects. All were native speakers of English and had normal or corrected vision.

Stimuli. The uppercase letters G, J, and R, and the Arabic numerals 2, 5, and 7 were taken from Letraset No.193 and mounted in double-glass slides which could be projected from either side to yield either normal or backward versions. Each character was mounted in six different angular orientations, in 60 -deg-steps from 0 to $300 \mathrm{deg}$ clockwise from the upright. There were, therefore, 72 different slides, made up of the two versions of each of the six characters in the six different angular orientations. These were arranged in a slide tray in a random sequence, with the restriction that no character, version, or orientation could appear more than three times in succession. The slides were rear projected onto a translucent screen about $1 \mathrm{~m}$ from the subject's eyes. Each character subtended a visual angle of $1 \mathrm{deg} 35 \mathrm{~min}$ along its longitudinal axis. A pinpoint of light continuously rear projected served as a reference point

Procedure. The subject sat at a table with his/her head in a chinrest which forced the head to remain in an upright position. A voice key was attached to the chinrest with a microphone about $2.30 \mathrm{~cm}$ from the subject's mouth. The experimenter explained that the operation of the voice key required fairly loud, abrupt vocalization, with no extraneous noises. Each character in each orientation and version was then displayed on a card, and the subject was asked to name it. Then, for the experimental session, the subject was told to fixate the reference point when the warning signal occurred, and to name each character as it appeared as quickly as possible. The warning signal was a tone lasting $500 \mathrm{msec}$, and the stimulus came on for $1 \mathrm{sec}$ beginning $500 \mathrm{msec}$ after offset of the tone. Each subject received the sequence of 72 slides twice, with a short break in between. The experimenter recorded the accuracy of the responses, and the latency between onset of the stimulus and operation of the voice key was automatically recorded on a printout timer.

\section{Results}

Errors. No subject made more than three errors in the 144 trials. When an error did occur, the latency for that trial was deleted and replaced with an estimate derived from the remaining "correct" latencies for that subject, testing block, and version, according to the formula

$$
\mathrm{RT}^{\prime}=\overline{\mathrm{R}} \overline{\mathrm{T}}_{\mathrm{c}}+\overline{\mathrm{R}} \overline{\mathrm{T}}_{\mathrm{o}}-\overline{\mathrm{R}} \overline{\mathrm{T}},
$$

where $\bar{R} \bar{T}_{c}$ is the mean for the character, $\bar{R} \bar{T}_{o}$ the mean 
for the orientation, and $\overline{\mathrm{R}} \overline{\mathrm{T}}$ the overall mean within the estimation sample (cf. Winer, 1971, p. 488).

Latencies. The latencies were subjected to analysis of variance. The characters differed significantly $[F(5,35)=18.21, p<.01]$; identification of the letter $R$ was the most rapid $(569 \mathrm{msec})$, followed in turn by the digit $5(614 \mathrm{msec})$, the letter $\mathrm{J}(628 \mathrm{msec})$, the digit 2 $(637 \mathrm{msec})$, the letter $\mathrm{G}(642 \mathrm{msec})$, and the digit 7 $(645 \mathrm{msec})$. In an earlier study of mental rotation of these characters (Corballis, Zbrodoff, \& Roldan, 1976), the ordering was the same except that there the letter $\mathbf{J}$ was in fifth position.

More importantly, there were significant main effects due to angular orientation $[\mathrm{F}(5,35)=6.98, \mathrm{p}<.01]$ and to version $[F(1,7)=48.60, p<.01]$. The interaction between orientation and version was also significant $[F(5,35)=3.04, p<.05]$, unless one adopts the reduced degrees of freedom $(\mathrm{df}=1 / 7)$ recommended by Greenhouse and Geisser (1959) for testing repeatedmeasurements effects. The interaction between testing blocks and orientations was also marginally significant $[F(5,35)=3.05, p<.05]$, as was the triple interaction between testing blocks, orientations, and versions $[F(5,35)=2.83, p<.05]$. The mean latencies for each combination of testing blocks, orientations, and versions are plotted in Figure 1.

Tests of simple main effects (Winer, 1971) were undertaken to determine the significance of the effect of angular orientation for each version within each testing block. According to these tests, angular orientation had a significant effect upon latency only within the first testing block, and then only for backward characters $[F(5,35)=10.49, p<.01]$. The effect of

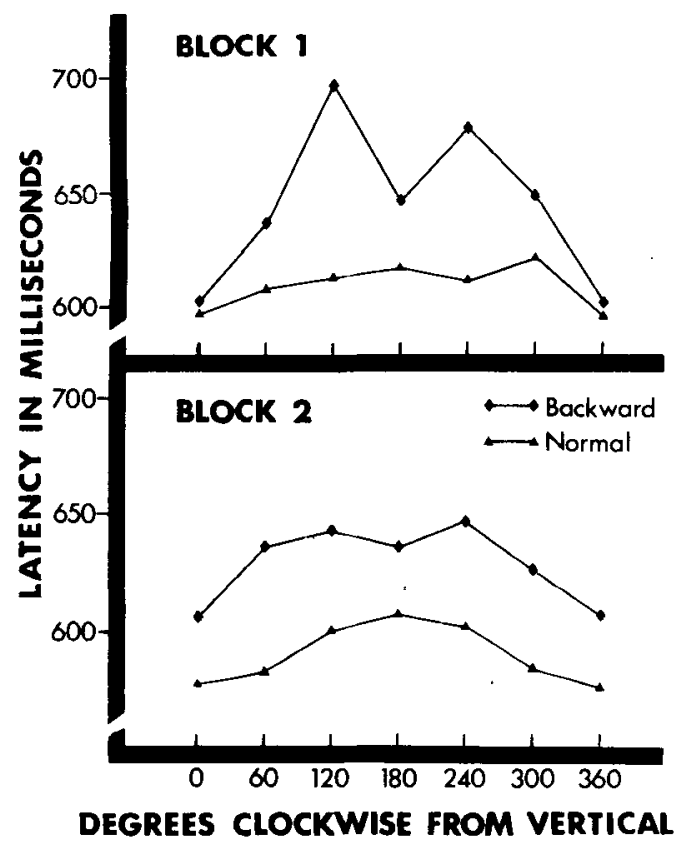

Figure 1. Mean latencies for naming normal and backward characters as a function of angular orientation in Experiment 1. orientation was thus insignificant for normal characters within the first testing block $[F(5,35)=.73]$ and insignificant for both backward and normal characters within the second testing block $[F(5,35)=2.01$ and 1.53 , respectively]. It is of interest to note, however, that the overall difference between latency to backward and latency to normal characters remained essentially constant, at about $40 \mathrm{msec}$, across the two testing blocks; the interaction between blocks and versions was negligible $[F(1,7)=.006]$.

The interaction between characters and orientations was marginally significant $[\mathrm{F}(25,175)=2.52, \mathrm{p}<.05]$, as was the triple interaction between characters, orientations, and versions $[F(25,175)=3.44, p<.01]$, although neither interaction is significant according to reduced degrees of freedom. The effect of angular orientation on latency to different characters can be roughly assessed by considering the maximum difference in latency between different orientations. For normal characters this difference was largest for the digit 7 $(108 \mathrm{msec})$, followed in order by $\mathrm{G}(89 \mathrm{msec})$, J $(57 \mathrm{msec}), 5(50 \mathrm{msec}), 2$ (31 msec), and R $(25 \mathrm{msec})$. For backward characters the order was $2(127 \mathrm{msec})$, $\mathrm{G}$ (123 msec), 5 (114 msec), 7 (98 $\mathrm{msec}), \mathrm{J}(58 \mathrm{msec})$, and $R(51 \mathrm{msec})$.

\section{Discussion}

The results show that the latency to name alphanumeric characters does depend to some extent on angular orientation, although the effect seems to be confined largely to backward characters and to dissipate with practice. However, even if we restrict our attention to the backward characters in the first testing block only (see Figure 1), we may note that the orientation function scarcely resembles those observed in experiments devised explicitly to investigate the mental rotation of the same set of characters (e.g., Cooper \& Shepard, 1973; Corballis et al., 1976). For one thing, the maximum difference in latency between adjacent orientations was only $58 \mathrm{msec}$, whereas group estimates of the time taken to mentally rotate a character through $60 \mathrm{deg}$ are about three times that interval. Second, there is a dip in the function for backward characters rotated $180 \mathrm{deg}$ from the upright, whereas the function for mental rotation is a maximum at this point. Finally, the functions tended to flatten out with practice, whereas the mental rotation functions reported by Cooper and Shepard (1973) were extremely resistant to practice effects.

Although our latency functions do not resemble mental rotation functions, it does not necessarily follow that the subjects never mentally rotated the characters in order to identify them. That is, it is conceivable that the subjects sometimes mentally rotated the characters. A subject may form an initial impression of the identity of a rotated character on the basis of the extraction of information that is independent of angular orientation 
(cf. Deutsch, 1955, 1962; Milner, 1974). In most cases, this impression may be sufficiently unambiguous to permit the subject to name the character without further processing, but the subject may occasionally choose to check the initial impression by mentally rotating the internal representation of the character to the upright, in order to match it against an internally generated template. One would indeed expect this checking strategy to decline in frequency as the subject grows more familiar with the somewhat restricted character set. Moreover, at least some of the differences between characters in the effects of orientation on latency can be explained in this way. For instance, a backward 2 might well be confused with a normal 5 , which perhaps explaines why the latencies to name the backward 2 were the most influenced by orientation. Further, the dip in the function for backward characters rotated $180 \mathrm{deg}$ might be explained by supposing that the subjects sometimes corrected an inverted character by mentally flipping it about its horizontal axis, in a plane orthogonal to its own plane. This would be particularly appropriate in the case of backward versions because the flip would restore normal parity, and thus lead to more rapid matching against an internally generated template of the normal version. We recognize, however, that this account is post hoc, and is indeed sufficiently flexible to explain almost any pattern of results. The main point we wish to emphasize is that the orientation functions, whatever explains them, need not entirely rule out mental rotation. However, mental rotation cannot have been more than an occasional strategy.

We may conclude, therefore, that identification does not normally require mental rotation. The results also showed that the latencies to identify backward characters were consistently longer than those to name the normal characters, and the difference was least when the characters were upright. Moreover, the effect of angular orientation was more pronounced in the case of backward than of normal characters, especially in the first testing block. These results raise something of a paradox, since they suggest that subjects must have some impression of whether a character is normal or backward before mentally rotating it. This conclusion seems to challenge the very rationale underlying Cooper and Shepard's (1975) task, in which it is assumed that mental rotation is necessary before an observer can tell whether a character is normal or backward; according to this rationale, the left-right orientation of a character is the last thing a subject knows about it.

It is of interest to note that the difference in latency to normal and backward characters was as great in the second testing block as in the first, even though the effects of angular orientation on the two versions were reduced in the second block. In the following experiment, we wished to determine whether the difference between versions would persist over more extended practice on an identification task.

\section{EXPERIMENT 2}

In this experiment, subjects were given extended practice on an identification task involving the same set of characters as in Experiment 1. Instead of naming the characters, however, each subject was given a particular target character and instructed to press one button whenever that character was displayed and the other button whenever any one of the other five characters was displayed.

\section{Method}

Subjects. Six undergraduates, four men and two women, all with normal or corrected vision, volunteered as subjects.

Stimuli and Apparatus. The stimuli were the same as in the first experiment. In this experiment, however, presentation of the stimuli, which were placed in a random-access projector, was controlled by a PDP-11 computer, which also recorded latencies. The computer selected the stimuli in random order according to specified frequencies; whenever a subject made an error, that trial was repeated at some later, randomly determined position in the sequence.

For this experiment, the voice key was replaced by a box with two buttons mounted on top of it. The box was placed on a table in front of the subject and positioned in such a way that the subject could comfortably press the buttons with the index and third fingers of the preferred hand.

Procedure. Each subject was assigned a different one of the six characters, which was to serve as the positive stimulus. Response probabilities were balanced so that on a given block of trials the positive character was presented five times in each orientation and version, whereas the negative characters were presented only once in each orientation and version. Thus, there were a total of 60 positive and 60 negative trials, excluding trials on which errors occurred, on each block. Each subject was tested over four blocks of trials, with a rest between blocks. $\mathrm{He} / \mathrm{she}$ worked with the same target character over the four blocks. The timing of the warning signal and character presentation on each trial was the same as in Experiment 1.

All subjects used the index finger of the preferred hand for positive responses and the third finger for negative responses.

\section{Results}

Errors. The subjects maintained a high rate of accuracy. The average error rate was $1.94 \%$ and was not obviously related to orientation, version, or decision.

Latencies. Latencies for correct decisions were subjected to analyses of variance. Since trials on which incorrect decisions occurred were repeated until the subject made a correct response, there was no correction necessary for incorrect responses. Separate analyses were carried out for positive and negative responses.

In the analysis of positive responses, the dependent variables were orientation, version, and block. Characters were confounded with subjects but we expected that this would have the effect of making the tests of significance, if anything, more conservative than they would otherwise have been. The analysis yielded no significant effects due to orientation or blocks, or the the interaction between them $(\mathrm{F}<1$ in each case). However, the latencies for backward characters were longer than those for normal characters, although the 
differences were significant only according to a directional test $[F(1,5)=5.43, .05<p<.10]$. This effect was independent of orientation and of blocks $(F<1$ for both interactions).

Five of the six subjects identified the target more rapidly when it was normal than when it was backward. The exception was the subject who was assigned the letter $\mathbf{J}$ as target, and his latency to backward $\mathbf{J s}$ was an average of $8 \mathrm{msec}$ shorter than his latency to normal ones. Among the five who responded more rapidly to the normal than to the backward versions, the latency differences, identified according to the character assigned as target, were $16 \mathrm{msec}(7), 30 \mathrm{msec}(5), 37 \mathrm{msec}$ (R), $44 \mathrm{msec}(\mathrm{G})$, and $114 \mathrm{msec}(2)$.

In the analysis of negative responses, the dependent variables were orientation, version, block, and character, which we treated as nested within subjects. There were no significant effects at all. Although latencies to backward characters were longer than those to forward ones, the difference was not significant $[F(1,5)=2.67$, $\mathrm{p}>.10]$.

The mean latencies are shown as a function of angular orientation and version in Figure 2.

\section{Discussion}

As in Experiment 1, the latency to identify backward characters was longer than the latency to identify normal ones, and the overall difference $(39 \mathrm{msec})$ was of the same order of magnitude. By way of contrast with Experiment 1, however, there was no evidence for any effect of angular orientation. This may have been due partly to the additional practice, although there was no significant effect of angular orientation even within the first testing block in this experiment. One reason for this discrepancy between the two experiments may have been that the target character was presented five times more frequently in this experiment than in Experiment 1 , so there was additional opportunity for practice

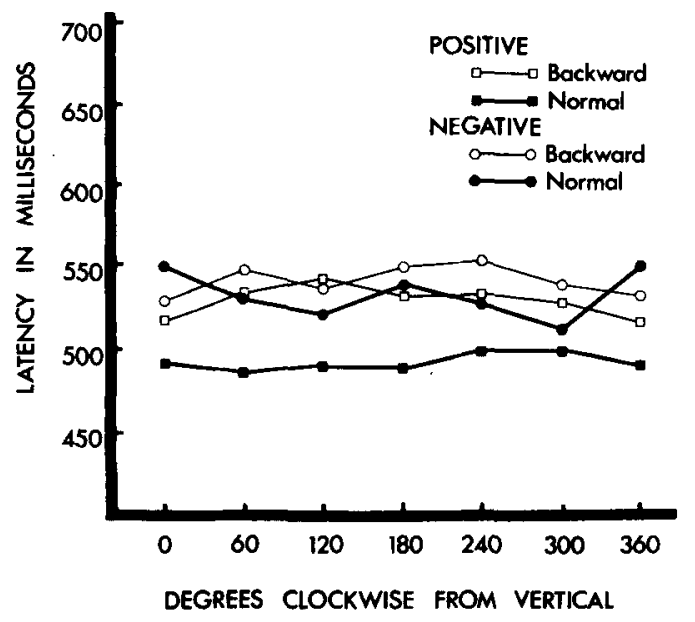

Figure 2. Mean latencies as a function of decision, version, and angular orientation in Experiment 2. even within the first testing block. The nature of the task also differed between the two experiments, and this may also have contributed. The task of identifying a single target might be considered simpler than that of naming all six characters, at least insofar as the subject need only establish a perceptual set for the single target. Indeed, one might speculate that this set may even have been nonverbal, or perhaps preverbal, when the target was a $\mathbf{J}$ or a 7 , since these can be viewed simply as a curved line (the $J$ had no bar across the top) and a simple angle, respectively. (This may explain why the effect of left-right orientation was least for these two characters.) But whatever the explanation for the different effects of angular orientation in the two experiments, the fact that the overall effect of left-right orientation remained approximately constant across experiments suggests that left-right orientation and angular orientation influence latency in different ways.

It may again seem paradoxical that the difference between normal and backward characters is evidently "registered" at a stage prior to any possible mental rotation, since the work of Cooper and Shepard (1973) suggests that subjects cannot actually decide whether a character is normal or backward unless they mentally rotate some internal representation of it to the upright. Even more strikingly, perhaps, Teichner and Krebs (1974) have reported that simple reaction times were shorter to normal than to backward letters despite the fact that the very identity of the letters was quite irrelevant to the task. Although one is forced to conclude that subjects compulsively encode information beyond the level required by the demands of the task, this result also implies that the left-right distinction is registered early in processing. One must suppose that this early registration is strong enough to influence latency but is too weak to permit subjects to make a confident decision about left-right orientation. We shall return to this point in the General Discussion.

\section{EXPERIMENT 3}

As we noted in the Introduction, Rock (1973) argued that it is necessary to assign an up-down axis to a pattern in order to recognize or identify it. Our results so far suggest that this assignment, if necessary at all, need not and usually does not involve mental rotation. However, Experiments 1 and 2 do not address themselves to the question of whether subjects need to know the angular orientation of a character before they can identify it. Experiment 3 was a preliminary attempt to address this question.

There were two different tasks. One was the identification task of Experiment 2: The subject was assigned a given target character in advance of each block of trials and told to press one of the two buttons whenever that character appeared, regardless of its orientation. The other task was an orientation task: The subject 
was assigned a given orientation in advance and told to press one of the two buttons whenever a character appeared in that orientation, regardless of the character. Stimulus probabilities were equated, so that the probability that a target would appear was equal for the two tasks; that is, for each task 12 of the 72 possible stimuli represented targets. We reasoned that if a subject must know the angular orientation of a character in order to establish its identity, then the latencies for the two tasks should be about the same and exhibit the same general properties. On the other hand, if identity is established first, one might expect latencies for the identification task to be generally shorter than those for the orientation task. As we shall see, these predictions are not entirely uncontaminated by other factors, but our hope was that relative performance on the two tasks would at least set some limits on the relative stages at which information about identity and about angular orientation is extracted.

\section{Method}

Subjects. The subjects were 24 undergraduate volunteers.

Stimuli and Apparatus. As in Experiment 1, the stimuli were arranged in random sequence in a slide tray and presented in order; it was not feasible to repeat individual stimuli when errors occurred. The response box used in Experiment 2 was also used in this experiment, but was connected to a printout timer rather than to the computer. The printout recorded which of the two buttons the subject pressed and the duration between onset of the stimulus and when a button was presented.

Procedure. Half of the subjects responded on the basis of the identity of the characters. On a given sequence of the 72 test stimuli, each subject was given a particular character $(G, J, R$, 2,5 , or 7 ) as a target and told to press one of the buttons (labeled "yes") with the index finger of the preferred hand if that target appeared on the screen, regardless of its angular or left-right orientation. If the character was other than the target, he/she was told to press the other button (labeled "no"). Each subject worked with each of the six characters as targets, and thus received the 72 stimuli six times, in different random orders. The orders in which the characters were assigned as targets was counterbalanced across subjects according to a digram-balanced Latin square, with two subjects assigned to each of the six orders.

The remaining 12 subjects responded on the basis of the orientation of the characters. The basic design was the same as for the identity group, except that on each sequence of 72 stimuli each subject was assigned a given orientation as a target and told to press the "yes" button whenever a character appeared on the screen in that orientation, regardless of which character it was and of whether it was normal or backward. The orientation was indicated before the trial sequence by an arrow drawn on a card, and the subjects were also shown examples of each character drawn in the target orientation. Each subject worked with each of the six orientations $(0,60,120$, 180,240 , or $300 \mathrm{deg}$, clockwise from vertical) in each of six presentations of the 72 stimuli, the order of assignment of orientations being counterbalanced across subjects.

Both groups saw exactly the same stimuli and, for both, 12 of the 72 stimuli were targets and 60 were nontargets. The subjects were given practice trials with extra slides to insure that they understood the task before the experimental trials began. They were told to respond as quickly as possible, consistent with high accuracy.

\section{Results}

Errors. Errors are tabulated in Table 1. Analysis of
Table 1

Mean Percentage of Errors for Each Orientation and Version in Identification and Orientation Tasks in Experiment 3

\begin{tabular}{llrrrrrr}
\hline & & \multicolumn{5}{c}{ Angular Orientation (Deg) } \\
\cline { 3 - 8 } Task & Version & \multicolumn{1}{c}{0} & \multicolumn{1}{c}{60} & 120 & 180 & 240 & 300 \\
\hline Identifi- & Normal & .69 & 1.39 & 1.16 & .69 & .46 & 1.39 \\
cation & Backward & 1.16 & .69 & 1.85 & .23 & 1.39 & 1.39 \\
Orienta- & Normal & 1.39 & 3.70 & 2.08 & 2.55 & 4.17 & 3.70 \\
tion & Backward & 8.10 & 4.63 & 4.63 & 2.55 & 4.86 & 8.80 \\
\hline
\end{tabular}

variance showed that there were significantly more errors for the orientation task than for the identification task $[F(1,22)=23.85, p<.001]$, and significantly more errors for backward than for forward characters $[F(1,22)=9.56, p<.01]$. There was also a significant interaction between task and version (forward vs. backward) $[F(1,22)=7.58, \mathrm{p}<.05]$, indicating that the version effect was smaller for the identification than for the orientation task, but this was probably due to a "floor effect" in the identification task and is of little interest.

Latencies. Latencies for incorrect responses were discarded from the analysis. In the case of positive responses, whenever a subject made an error the latency was estimated from the latencies of his/her remaining correct responses within the same block of trials according to a procedure comparable to that specified in Experiment 1. In the case of negative responses, latencies were simply averaged over correct responses for each orientation and version in each block.

Analyses of variance were first carried out separately for each task. In each case there were five independent variables: decision (positive vs. negative), character, angular orientation, version, and order. Only the last was a between-subjects variable.

For the identification task, there were significant main effects due to decision $[\mathrm{F}(1,6)=27.22, \mathrm{p}<.01]$, angular orientation $[F(5,30)=10.56, p<.001]$, and version $[F(1,6)=11.45, p<.05]$. There were also significant interactions between decision and angular orientation $[F(5,30)=5.81, p<.001]$ and between decision and version $[F(1,6)=11.27, p<.05]$. Figure 3 shows the mean latencies for each decision, version, and angular orientation. It is clear that the effects of angular orientation and of version are less marked for negative than for positive decisions. The interaction between version and angular orientation was not significant $[F(5,30)=2.30, p>.05]$. In particular, there was no dip in latency of identifying backward characters at 180 deg comparable to that observed in Experiment 1; paradoxically, there was a slight dip in the orientation function at $180 \mathrm{deg}$ for forward characters. The fact that angular orientation influenced latencies is also in contrast with the results of Experiment 2. The discrepancy may be due in part to the different stimulus and response probabiliteies; only one-sixth of the stimuli were targets in the present experiment, compared with one-half in Experiment 2. It may also have 
had to do with the fact that the target changed from block to block in the present experiment.

For the orientation task, there were significant main effects due to decision $[F(1,6)=116.97, p<.01]$, character $[F(5,30)=18.08, p<.001]$, angular orientation $[F(5,30)=4.91, p<.01]$, and version $[F(1,6)=$ $73.85, \mathrm{p}<.001]$. Character interacted significantly with both decision $[F(5,30)=9.60, p<.001]$ and version $[F(5,30)=5.25, p<.01]$. There was a sig. nificant interaction between decision and version $[F(1,6)=23.61, p<.01]$, but neither variable interacted significantly with angular orientation, nor was the triple interaction significant. Mean latencies for each decision, version, and angular orientation are also shown in Figure 3.

In order to highlight the differences in the pattern of results between the two tasks, we also computed an analysis of variance with task as an independent variable. The order of testing for different target characters or orientations appeared to be unimportant within each task, and was deleted from the analysis. Only those effects involving task are of interest. First, latencies were significantly shorter overall in the identification task than in the orientation task $[F(1,22)=35.71, p<.01]$. Task also interacted significantly with decision $[F(1,22)=23.23, p<.01]$ and with version $[F(1,22)=$ $9.53, \mathrm{p}<.01]$. The interaction of task with angular orientation $[F(5,110)=2.77]$ and the triple interaction between task, angular orientation, and version $[F(5,110)=2.38]$ are significant $(p<.05)$ according to conventional degrees of freedom but not according to the conservative degrees of freedom $(1 / 22)$ recommended for testing repeated-measurements effects (Winer, 1971), and must therefore be considered marginal. The triple interaction between tasks, decision, and version was highly significant $[F(1,22)=15.60, p<.01]$.

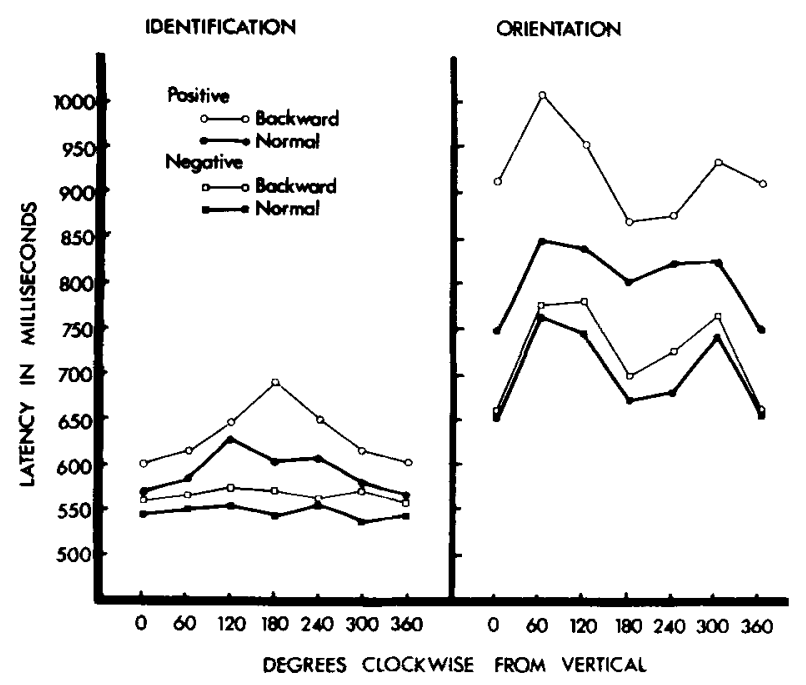

Figure 3. Mean latencies as a function of decision, version, and angular orientation for the identification task (left panel) and for the orientation task (right panel) in Experiment 3.
The nature of all of these effects can be seen in Figure 3 .

There were also significant interactions between task and character $[F(5,110)=13.21, \mathrm{p}<.001]$ and be. tween task, character, and decision $[F(5,110)=7.47$, $\mathrm{p}<.001]$. The product moment correlation between the tasks across the six characters was -.175 , indicating that the factors governing relative speed of identifying the characters were different from those governing relative speed of judging their orientations. The correlation between the identification task and the naming task of Experiment 1 was .335 , while that between the orientation task and the naming task was -.154 .

\section{Discussion}

It is clear that it took much longer to decide on the orientations of the characters than to decide on their identities. Moreover, the two tasks produced different patterns of results. These differences cannot be attributed to speed-accuracy tradeoff, since there were many more errors in the orientation task than in the identification task. Tentatively, we conclude that identification of an alphanumeric character did not require the observer to establish its orientation, at least within the limited context of this experiment. Orientation is determined subsequent to identity.

However, this conclusion must remain tentative, for although the two tasks were matched in terms of stimulus and response probability, there was one respect in which they could not be matched. The judgment itself may have been more difficult in the case of the orientation task than in the case of the identification task. Orientation is a continuous, noncategorical dimension, while identity is a highly overlearned, categorical one. We suspect that the elevated latencies for the oblique orientations in the judgments of orientation (see Figure 3) have been due partly to the ill-defined quality of the mental set for a particular oblique-the so-called "oblique effect" (Appelle, 1972)-although it is not clear why some oblique orientations (e.g., $240 \mathrm{deg}$ ) yielded quite short latencies, while others (e.g., $60 \mathrm{deg}$ ) yielded much longer ones. However, one might expect any judgmental difficulty to be fairly negligible when the target orientation is the upright, since we are highly familiar with the upright in the everyday world. It is noteworthy, therefore, that the average latency to decide that an upright character was upright $(742 \mathrm{msec})$ was significantly longer than the average latency to identify even an inverted character $(604 \mathrm{msec})$ [ $\mathrm{t}(22)=$ $2.31, \mathrm{p}<.05]$. Thus, even if there is an extra judg. mental component in the latency to establish orientation, we suspect that our original conclusion holds: namely, that subjects can generally identify a character before they know its orientation.

A more secure conclusion, perhaps, is that knowledge about orientation does not require mental rotation, as Rock (1973) seems to have implied, but must precede mental rotation. The latencies in the orientation task 
were shorter than those which typically occur in the task devised by Cooper and Shepard (1973), in which subjects must decide whether the characters are normal or backward. For instance, Corballis et al. (1976) reported latencies ranging from about $890 \mathrm{msec}$ for upright characters to about $1,370 \mathrm{msec}$ for inverted characters in the Cooper-Shepard task, under conditions comparable to those of the present study: The actual stimuli and apparatus were the same, but the response probabilities were different. The mean latencies for the orientation task in the present experiment ranged from $742 \mathrm{msec}$ for upright characters to $847 \mathrm{msec}$ for characters tilted $60 \mathrm{deg}$. Consequently, the data of the present experiment, taken together with those of Corballis et al. (1976), are consistent with the interpretation that subjects first establish the identity of a character, then determine its angular orientation, and, finally, mentally rotate an internal representation to the upright in order to determine whether the character is normal or backward.

\section{GENERAL DISCUSSION}

One conclusion which emerges fairly clearly from the present experiments is that identification does not normally require mental rotation, at least in the case of the familiar alphanumeric characters used in our experiments. This is not to say that the process of identification is independent of angular orientation. Identification latency did vary with angular orientation in Experiments 1 and 3. However, it is not clear from our experiments what underlay these orientation effects, or why they differed from experiment to experiment; the important point is that the effects of orientation did not at all resemble what one would expect had the subjects consistently adopted a strategy of mental rotation.

Speculatively, let us suppose that the brain first induces a description of a shape which is more or less independent of its angular orientation. Such a description is essentially a representation of relations internal to the shape, and not of its relations to other shapes or to the visual field as a whole; it may depend on the extraction of features (e.g., Deutsch, 1955, 1962; Milner, 1974) or perhaps on more global processes (e.g., Pollen, Lee, \& Taylor, 1971). In some cases this description may be sufficient for the observer to identify and label the shape, particularly if there is only a limited set of highly familiar shapes from which to choose and if the distinction among them does not depend on knowing their angular orientations. These conditions prevailed in the present experiments. In Experiments 1 and 3, the subjects may have sometimes processed the information further in order to identify the character, even though it may not have been necessary to do so. In some cases (not tested in the present experiments) we must suppose that information about shape per se would be insufficient to permit identification. For instance, the distinctions among the lowercase letters $b, d, p$, and $q$, or between a square and a diamond, clearly depend critically on knowledge about orientation as well as about shape.

Having established a description of a shape which is largely independent of its angualr orientation, the observer may then infer or assign an up-down axis; that is, the shape is now seen in a particular orientation relative to its normal or "upright" orientation. If the observer already knows what the shape is, he or she can then pick out its "top" and "bottom" and thus infer its angular orientation. This, of course, implies reference to location information which is initially independent of information about shape per se (cf. Schneider, 1969). In some cases, the perceived orientation of the shape may be "assigned" rather than inferred (cf. Rock, 1973). For instance, a tilted square may be seen as a diamond if one chooses to place the up-down axis through opposite corners, or as a square if one sees two of the parallel sides as "top" and "bottom"; again, an uppercase M may be seen as an inverted $W$ simply by reversing the direction of the assigned axis. Although the process of establishing an up-down axis may rightly be viewed as a "cognitive act," we doubt that it involves mental rotation, as Rock (1973) suggested. For one thing, the results of Experiment 3 suggested that information about the orientation of an alphanumeric character is available before the observer has had time to perform a mental rotation. But, in any case, as we pointed out in the Introduction, it is logical to supppose that knowledge about angular orientation is a prerequisite of mental rotation rather than a consequence of it.

Once the angular orientation of a shape is established, the observer can make further distinctions. For instance, he or she can now label the lowercase letter $d$ as distinct from a $p$, even though the distinction may involve the essentially arbitrary assignment of an up-down axis (e.g., if the $\mathrm{d}$ is rotated $90 \mathrm{deg}$, it may be seen with equal plausibility as a p). The point is that the assignment of the axis, however arbitrary, further disambiguates the percept. However, the observer may still have difficulty distinguishing a $\mathrm{d}$ from $\mathrm{a} b$ at this level of analysis. As Cooper and Shepard have shown, discrimination of mirror-image forms apparently requires mental rotation of some internal representation of a given form to the standard upright, presumably for matching against some internally generated template (Cooper, 1975; Cooper \& Shepard, 1973).

There is one paradox which remains, however: Latency to identify backward characters was consistently longer than latency to identify normal characters. This appeared to be the case even in Experiment 2, in which latency was independent of angular orientation. Thus, there seems to be a sense in which the left-right orientation "registers" early in perceptual processing, even though the last thing an observer "knows" about a 
character is whether it is normal or backward. That left-right orientation makes some impression on early identification is at odds with the feature-extraction theories of shape receognition proposed by Deutsch (1955, 1962) or Milner (1974), since these theories predict invariance with respect to both angular orientation and mirror reflection.

An alternative approach which may be more easily reconciled with the present data is that there is a process of mirror-image generalization, perhaps involving homotopic transfer of memory traces between the two halves of the brain, such that registration of a pattern leaves memoiy traces both for the pattern itself and for its left-right mirror image (Achim \& Corballis, 1977; Corballis \& Beale, 1970, 1976). Such a process would serve an adaptive function in the everyday world, since it would enable us to recognize patterns (such as faces in profile) regardless of their left-right orientation. However, alphanumeric characters are somewaht exceptional in that they nearly always appear in their normal versions and are seldom seen left-right reversed. The reversed traces would therefore be laid down more weakly than the veridical ones, since they would depend largely on interhemispheric transfer rather than on direct input. Cerebral lateralization may also play a role in determining the relative strengths of veridical and reversed traces. Bradshaw, Bradley, and Patterson (1976) have shown that latency to identify normal letters is typically shorter if they are presented in one visual field (usually the right) than in the other, but that this difference is diminished or even reversed for backward letters. They interpret this as evidence for interhemispheric mirror-image reversal in the establishment of memory for letters, with the veridical trace established most strongly in the dominant cerebral hemisphere. Similarly, we may suppose that in our subjects the normal versions had access to stronger traces than did the backward versions, and were thus identified with shorter latency. However, we must also suppose that this difference in strength is insufficient to permit a confident decision about whether a given character is actually normal or backward, and that subjects must resort to mental rotation in order to make this decision.

So far, our account has dwelt on the extraction of different kinds of information from the stimulus input. An alternative, though not necessarily contradictory, approach might emphasize rather the role of the subjects' familiarity with the stimuli. For instance, in the previous paragraph we implicitly recognized that the latency differential between normal and backward characters depends fundamentally on the fact that our experience in the everyday world is largely confined to normal versions. That we can readily identify backward characters, albeit with slightly increased latency, was attributed to a built-in mechanism for mirror-image generalization. It is possible, of course, that identification of backward characters does depend in part on direct experience with reversed forms (as in a mirror, for example), although this can scarcely explain why children so readily confuse mirror-image forms (Corballis \& Beale, 1976).

Another argument against the approach we have taken is that experience may play a critical role within the context of a given experimental task. For instance, it might be argued that the reason identification was independent of angular orientation under some conditions was simply that the subjects learned to identify each rotated character specifically, without concern for the invariance underlying different rotations of the same character. Although this may have been true up to a point, there are several arguments which suggest that it is not the whole story. In Experiment 2, in contrast with Experiments 1 and 3, latencies to identify the characters were independent of angular orientation even in the first testing block, suggesting that the degree of angular orientation has more to do with the nature and parameters of the task than with practice per se. Second, Cooper and Shepard (1973) gave their subjects extensive practice, but decisions about version (normal vs. backward) remained heavily dependent upon orientation, even when the subjects knew in advance what the orientation would be, suggesting that the subjects were unable to learn the specific stimulus configurations associated with each version. Finally, in Experiment 2, practice seemed to have no effect on the latency differential between normal and backward versions. However, we cannot rule out the possibility that the discrimination of different versions is intrinsically more difficult than the discrimination of different characters, and that it was therefore easier for subjects to learn to identify the rotated characters independently of version in each specific orientation than it was for them to identify their versions.

As a final comment, we should note that our analysis is speculative and may be restricted in generality to highly familiar codable shapes such as alphanumeric characters or simple geometric forms. Different principles may underlie the recognition of more complex or less categorical patterns, such as faces or the relatively unfamiliar nonsense forms studied by Olshansky (Note 1).

\section{REFERENCE NOTE}

1. Olshansky, P. Phenomenal orientation in form perception. Unpublished paper, Yeshiva University, 1966 (cited by Rock, 1973).

\section{REFERENCES}

Achim, A., \& Corballis, M. C. Mirror-image equivalence and the anterior commissure. Neuropsychologia, 1977, 15, 475-478.

Appelle, S. Perception and discrimination as a function of stimulus orientation: The "oblique effect" in man and animals. Psychological Bulletin, 1972, 78, 266-278. 
Bradshaw, J., Bradley, D., \& Patterson, K. The perception and identification of mirror-reversed patterns. Quarterly Journal of Experimental Psychology, 1976, 28, 221-246.

COOPER, L. A. Mental transformations of random two-dimensional shapes. Cognitive Psychology, 1975, 7, 20-43.

Cooper, L. A., \& Shepard, R. N. Chronometric studies of the rotation of mental images. In W. G. Chase (Ed.). Visual information processing. New York: Academic Press, 1973.

Corballis, M. C., \& Beale, I. L. Bilateral symmetry and behavior. Psychological Review, 1970, 77, 451-464.

Corballis, M. C., \& Beale, I. L. The psychology of left and right. Hillsdale, N.J: Lawrence Erlbaum, 1976.

Corballis, M. C., Zbrodoff, J., \& Roldan, C. E. What's up in mental rotation? Perception \& Psychophysics, 1976. 19. 525-530.

DeutsCh, J. A. A theory of shape recognition. British Journal of Psychology, 1955, 46, 30-37.

Deutsch, J. A. A system for shape recognition. Psychological Review, 1962, 69, 492-500.

Greenhouse, S. W., \& Geisser, S. On methods in the analysis of profile data. Psychometrika, 1959, 24, 95-112.
Kolers, P. A., \& Perkins, D. N. Orientation of letters and their speed of recognition. Perception \& Psychophysics, $1969,5,275-280$.

Milner, P. M. A model for visual shape recognition. Psychological Review, 1974, 81, 521-535.

Pollen, D. A., Lee, J. R., \& Taylor, J. H. How does the striate cortex begin the reconstruction of the visual world? Science, 1971 173, 74-77.

Rock, I. Orientation and form. New York: Academic Press, 1973.

Schneider, G. E. Two visual systems. Science, 1969, 163, 895-902.

ShePARD, R. N., \& METZleR, J. Mental rotation of threedimensional objects. Science, 1971, 171, 701-703.

TEIChNER, W. H., \& KREBS, M. J. Laws of visual choice reaction time. Psychological Review, 1974, 81, 75-98.

WINER, B. J. Statistical principles in experimental design. New York: McGraw-Hill, 1971.

(Received for publication July 14, 1977; accepted November 7,1977 .) 